\title{
FACTORES AMBIENTAIS E PSICOSSOCIAIS DA AVALIAÇÃO DO RISCO DE FURTO POR CARTEIRISTA: O CASO DE DOIS LOCAIS EM LISBOA ${ }^{1}$
}

\author{
Elena Sautkina ${ }^{2}$ \\ Clara António ${ }^{3}$ \\ Hugo Caseira ${ }^{4}$ \\ Filipe Correia ${ }^{5}$ \\ Dimitris Dimitriou 6 \\ Cláudia Grijó ${ }^{7}$ \\ Ana Loureiro ${ }^{8}$ \\ Rita Morais ${ }^{9}$
}

Resumo: É do conhecimento comum que o furto por carteirista é um tipo de crime que se encontra em ambiente urbano. Baseada em factores ambientais e psicossociais de representações espaciais de segurança/insegurança, previamente identificados, a presente investigação pretendeu explorar como este tipo de factores podem afectar as avaliações das pessoas, no que se refere ao risco de serem assaltadas por carteiristas. Neste sentido, foram considerados dois locais em Lisboa, diferindo principalmente nas suas características ambientais, físicas e sociais. Os resultados mostram que fortes ligações sociais num local podem reveler-se muito importantes para a avaliação do risco de furto por carteirista, afastando e tornando menos importante as características ambientais físicas, enquanto que, nos locais de maior heterogeneidade social, onde as ligações sociais são fracas, o ambiente físico torna-se consequentemente mais importante para as percepções de segurança.

Palavras-chave: furto por carteirista, avaliação do risco, ligações sociais, representação espacial.

\footnotetext{
${ }^{1}$ A correspondência relativa a este artigo deverá ser remetida para Elena Sautkina, Departamento de Psicologia Social e das Organizações, Edifício ISCTE, Avenida das Forças Armadas, 1649-026 Lisboa, ou via e-mail para elena.sautkina@iscte.pt. Este estudo foi realizado no contexto do workshop: Sautkina, E., \& Amador, S. (2005). Spatial and Psychosocial Factors of Crime Victimization in Urban Environment. Escola de Verão em Psicologia Ambiental. ISCTE, Lisboa, 12-18 de Julho. Agradecemos a Sofia Farinha e Filipa Jácome a sua participação na recolha dos dados.

2,3,5,9 ISCTE, Lisboa.

${ }^{4}$ Universidade do Minho.

${ }^{6}$ City University of London, Reino Unido.

${ }^{7}$ Universidade de Coimbra.

${ }^{8}$ Universidade Lusófona de Humanidades e Tecnologias.
}

PSICOLOGIA, Vol. XXI (2), 2007, Edições Colibri, Lisboa, pp. 79-97. 
Environmental and Psychosocial Factors of Pick-pocketing Risk Evaluation: The Case of Two Sites in Lisbon (Abstract): It is in common knowledge that pick-pocketing is a type of crime encountered in urban environments. Based on previously identified environmental and psychosocial factors of spatial representations of security/insecurity, the current research aimed at exploring environmental factors that could affect people's evaluations, as regards the risk of being pick-pocketed. With this purpose, two sites in Lisbon differing mainly in their physical and social environmental features were considered. Results show that strong social bonds within a place may appear very important for evaluation of pick-pocketing risk, putting aside and making less important physical layout, whereas in socially heterogeneous places, where social bonds are weaker, physical environment becomes consequently more important for perceptions of safety.

Key-words: pick-pocketing, risk evaluation, social bonds, spatial representation.

\section{Introdução}

No contexto do crescimento das cidades, o problema da delinquência, ampliado por factores ambientais, é algo bastante frequente. Assim, o crime contra o património ocupa, geralmente, posições elevadas nas estatísticas de criminalidade nas cidades. Em Lisboa, representa $67,8 \%$ do total de crimes cometidos (Relatório de Segurança Interna, 2002). A pesquisa sobre a relação entre crime e ambiente tem-se focado nos factores ambientais de alguns crimes contra o património (Brantingham \& Brantingham, 1993), dedicando menos atenção a outros. Também, os factores psicossociais desta categoria de crime raramente têm sido objecto de estudo.

O furto por carteirista, um dos crimes a que os cientistas têm dado pouco relevo (Sautkina, 2004), pode ser definido como um furto sub-reptício, não violento, de objectos de sacos ou de bolsos de um indivíduo. A vítima de furto por carteirista (num estado de lucidez) não tem consciência do furto no momento em que este está a ser cometido, dado que os carteiristas agem de um modo extremamente discreto. De referir também que o furto por carteirista é um crime urbano, na medida em que o ambiente urbano apresenta diversos factores que facilitam a actividade dos carteiristas, tais como a elevada densidade social e física, ou diversas situações favorecedoras de distracção que causam uma sobrecarga cognitiva (Sautkina, 2004). O furto por carteirista é um dos crimes mais frequentes em Lisboa (Tabela 1). 
Tabela 1 - Quatro crimes que se destacam pelo elevado número de participações registadas (Relatório de Segurança Interna, 2002)

\begin{tabular}{|lcccc|}
\hline \multicolumn{1}{|c}{ Distrito de Lisboa } & Ano 2001 & Ano 2002 & $\begin{array}{c}\text { Diferença } \\
\mathbf{2 0 0 1 / 0 2}\end{array}$ & $\begin{array}{c}\text { Variaçäo } \\
\mathbf{2 0 0 1 0 2}\end{array}$ \\
\hline \multicolumn{1}{|c|}{ TOTAL GERAL } & 98.822 & 102.695 & 3.873 & 3,9 \\
\hline Furto em veículo motorizado & 16.304 & 17.703 & 1.399 & 8,6 \\
\hline Ofensa à integridade fisica voluntária simples & 8.429 & 9.297 & 868 & 10,3 \\
\hline Furto de veículo motorizado & 6.580 & 8.132 & 1.552 & 23,6 \\
\hline Furto por carteirista & 7.836 & 6.775 & -1.061 & $-13,5$ \\
\hline
\end{tabular}

O objectivo deste artigo é mostrar como as pessoas comuns percebem o risco de furto por carteirista, no ambiente urbano. Nas secções que se seguem são referidos alguns princípios teóricos e resultados de pesquisas anteriores em que o estudo se baseia. Também são dadas descrições dos dois locais em Lisboa (Alfama e Praça do Comércio), onde se desenrolou a nossa investigação.

\section{Factores ambientais e psicossociais do medo do crime e do sen- timento de segurança}

Medo de crime (fear of crime) é um receio de ataques e das suas consequências: prejuízo e lesões causadas à integridade psicológica e/ou física do indivíduo (Brantingham \& Brantingham, 1997).

O medo do crime não está directamente relacionado com as taxas reais da criminalidade. Este fenómeno tem sido denominado "paradoxo espacial" (Pain, 1993). Um número significativo de investigações evidenciam que as distribuições espaciais do medo do crime e as dos crimes propriamente ditos podem não coincidir (Taylor \& Hale, 1986; Brantingham \& Brantingham, 1991; Nasar \& Fisher, 1993; Esteves, 1999). Deste modo, os locais onde se registam objectivamente taxas elevadas de crime não são, necessariamente, aqueles onde a população se sente insegura (Donnelly, 1988), e vice versa, os crimes podem ser cometidos em lugares onde as pessoas se sentem seguras. A existência do "paradoxo espacial" tem-se verificado para o furto por carteirista (Sautkina, 2004).

Este fenómeno tem um carácter multifactorial. As características ambientais que contribuem para o medo do crime nem sempre correspondem àquelas que objectivamente contribuem para os actos criminosos, por- 
que o ponto de vista da potencial vítima é diferente do do criminoso (Chaguiboff, 1992). Além disso, o medo do crime está relacionado com os significados sociais que as características ambientais têm para as pessoas (Brantingham \& Brantingham, 1991): certos lugares, por causa da presença de indícios ambientais geradores de medo do crime, tornam-se estigmatizados e ficam conotados como "sítios perigosos".

Determinadas características ambientais (físicas e sociais) tendem a produzir medo do crime e sentimento de segurança.

Os indivíduos necessitam de vigiar a situação para que se possam adaptar a ela e controlá-la de uma forma objectiva ou subjectiva (Hall, 1966). Appleton (1975) sugeriu que as pessoas percepcionam os lugares de acordo com o grau de perspectiva (possibilidade de visão) e a possibilidade de refúgio (i.e., de abrigo, protecção). Investigações posteriores (Nasar \& Fisher, 1993; Loewen et al., 1993) enfatizam o facto de que os indivíduos se sentem mais seguros em lugares que oferecem perspectiva e refúgio físico suficiente (i.e., saídas escapatórias que permitam a uma potencial vítima fugir ao perigo), enquanto que ruelas longas, ruas escuras, com poucos pontos de fuga, tendem a provocar medo do crime.

De acordo com a hipótese "janelas partidas" (Wilson \& Kelling, 1982), indícios de desorganização e deterioração podem ser percepcionados como símbolos de declínio da ordem social e, assim, conduzir ao medo do crime (Nasar, 2000). Indícios de desorganização social (embriaguez pública, venda e consumo de drogas, prostituição, sem-abrigo) é a mais manifésta. Contudo, indícios de desorganização física (graffiti, vandalismo, lixo, má manutenção das ruas e edifícios) podem transmitir "poderosas mensagens de anarquia" (Perkins et al., 1993; Wilcox et al., 2003) e são percepcionadas como menos perdoáveis (Félonneau \& Lannegrand-Willems, 2005). Os indícios de desorganização podem ser interpretados em termos de controlo social formal e/ou informal ineficiente. O controlo social informal assegura conformidade com as normas sociais e implica a presença de péssoas que (de uma maneira não oficial) procurem manter o respeito pelas regras sociais (ver Taylor, 1987). O controlo social formal assegura o respeito pelas leis oficiais, através de representantes institucionais: polícia e vigilância (Kelling \& Moore, 1981). Os sinais de controlo social eficiente dão um sentimento geral de protecção e segurança, enquanto que as percepções da sua ineficácia podem conduzir ao medo do crime.

A heterogeneidade social está relacionada com o medo do crime, porque pode provocar um sentimento de incerteza, medo de estranhos, da "diferença", pois os hábitos e comportamentos dos outros podem mostrar-se difíceis de interpretar (Merry, 1981). 
Estudos anteriores de Sautkina (2004) demonstraram a importância dos factores listados para a avaliação do risco de furto por carteirista, em Paris e Moscovo, mostrando, assim, a sua natureza complexa.

\section{Descrição dos locais: Alfama e Praça do Comércio}

O presente estudo foi realizado em dois locais: Alfama e Praça do Comércio. Alfama é um dos mais típicos e emblemáticos bairros de Lisboa e, por isso, muito visitado por turistas, sendo ainda um dos núcleos originais da cidade. As ruas estreitas e sinuosas caracterizam este espaço urbano complexo e denso. A população actual provém dos fluxos de êxodo rural e, apesar de alguma heterogeneidade social, a maioria aufere baixos rendimentos e tem reduzidas habilitações escolares. É também possível observar alguns estilos de vida associados à marginalidade e criminalidade. De facto, Alfama tende a estar associada à pequena criminalidade, nomeadamente o furto por carteirista. Devido ao facto de as famílias viverem aqui há bastante tempo, o bairro desenvolveu redes densas familiares e de vizinhança (Silva, 2000). Por outro lado, as festas populares como as "marchas populares" e os "arraiais" reflectem a forte identidade de Alfama (Costa, 2003). Devido à sua degradação, este bairro encontra-se num processo de requalificação urbana, apesar de sinais de pobreza, falta de manutenção e lixo serem ainda visíveis.

A Praça do Comércio é uma das maiores praças da Europa, ampliada ainda pela presença do grande estuário do rio. Situam-se perto desta praça o porto de Lisboa e a estação ferroviária, bem como a estação marítima, destinada à ligação e transporte entre as duas margens do rio. A praça tem igualmente bastantes paragens de autocarro e de eléctrico, algumas delas destinadas ao turismo (Praça do Comércio. O seu a seu dono, 1998). Durante o dia, este é um local agitado, com a passagem de pessoas dos subúrbios e da outra margem do rio, turistas, automóveis e autocarros que atravessam a praça. Esta grande actividade contrasta com a baixa densidade social à noite e aos fins-de-semana. Durante os últimos anos, a zona em frente ao rio tem sido ocupada com obras do metropolitano.

Neste estudo, pretendemos verificar se as avaliações do risco de furto por carteirista correspondem às taxas reais deste crime em Alfama e na Praça do Comércio. Esperamos que os participantes avaliem como elevado o risco de furto por carteirista em Alfama e reduzido na Praça do Comércio, baseado nas suas percepções das características socioambientais de cada local, que podem ser consideradas quase como opostas em termos de indícios de desorganização, perspectiva e saídas escapatórias, heterogeneidade étnica (como vimos anteriormente, níveis elevados destas características 
estão relacionados com medo do crime). Ao mesmo tempo, e de acordo com informações obtidas na Esquadra do Turismo de Lisboa, a Praça do Comércio é conhecida pela sua elevada taxa de furtos por carteirista, enquanto que Alfama é conhecida por níveis reduzidos deste crime. Além disso, a zona da Baixa da cidade é uma das áreas de Lisboa com níveis de crime consideráveis, associados ao emprego e comércio, os quais são características relevantes desta zona (Nicolau, 1994). Assim, esperamos encontrar o fenómeno de "paradoxo espacial" em relação à avaliação do risco de furto por carteirista, nestes dois locais.

\section{Método}

\section{Participantes e procedimento}

Em ambos os locais foram realizadas entrevistas semiestruturadas a transeuntes, com o objectivo de perceber como é que avaliam o risco de furto por carteirista nesses locais, bem como explicam estas avaliações do risco. Desta forma, escolhemos estudar as avaliações do risco de furto por carteirista in situ, no contexto natural das actividades rotineiras dos respondentes. Foram entrevistados 45 participantes em Alfama e 46 na Praça do Comércio. Em ambos os locais, as amostras são equilibradas, em termos de sexo e de categorias etárias. Para além disso, foi também recolhida informação acerca do local de residência dos participantes.

Foi pedido aos participantes que considerassem a probabilidade e o grau em que o local pode favorecer o furto por carteirista, usando uma escala de Likert (1 - "Muito baixo"; a 5 - "Muito elevado"). Posteriormente, era solicitado aos sujeitos que explicassem a sua avaliação do risco, usando uma lista de categorias opostas, baseada em investigações anteriores (Sautkina, 2004), como mostra a Tabela 2.

\section{Descrição do método de análise dos dados}

Uma vez que as frequências e percentagens não são suficientes para resumir as associações nas tabelas de contingência, recorremos a dois níveis de análise: descritiva (análise das taxas de associação) - de forma a resumir as associações existentes entre as modalidades das variáveis ao nível da nossa amostra; e inferencial (análise inferencial Bayesiana) - para generalizar os resultados obtidos para a população global.

$\mathrm{O}$ rácio de desvio da independência tem sido utilizado com o nome de "taxa de associação" (taux d'association) - (ver Rouanet et al., 1987). Uma taxa de associação é igual a zero quando as duas modalidades são independentes. De salientar que, por um lado, o valor de associação negati- 
va mínima é -1, mas, por outro lado, não existe um valor máximo, quando a taxa de associação é positiva.

Tabela 2 - Categorias das explicações verbais face à avaliação do risco

\begin{tabular}{|c|c|}
\hline Categoria & Definição nas tabelas \\
\hline $\begin{array}{l}\text { Existem muitas pessoas aqui: } \\
\text { isso "ajuda" os carteiristas }\end{array}$ & Muitas pessoas \\
\hline Não existem muitas pessoas aqui & Poucas pessoas \\
\hline As pessoas aqui são semelhantes a mim & Pessoas semelhantes a mim \\
\hline As pessoas aqui são diferentes de mim & Pessoas diferentes de mim \\
\hline $\begin{array}{l}\text { Aqui não existe deterioração. Este local é esteticamente } \\
\text { agradável e bem-cuidado }\end{array}$ & Ausência de deterioração \\
\hline $\begin{array}{l}\text { Aqui existe bastante deterioração } \\
\text { (graffiti, lixo, pouco cuidado) }\end{array}$ & Deterioração \\
\hline Passividade das outras pessoas aqui & Passividade dos outros \\
\hline Reactividade das outras pessoas aqui & Reactividade dos outros \\
\hline $\begin{array}{l}\text { Este local é bastante vigiado (elevada presença } \\
\text { da polícia/segurança) }\end{array}$ & O local é vigiado \\
\hline $\begin{array}{l}\text { Este lugar não é vigiado (reduzida presença } \\
\text { da polícia/segurança) }\end{array}$ & O local não é vigiado \\
\hline $\begin{array}{l}\text { Aqui existem muitos turistas e lojas. Isso significa } \\
\text { dinheiro e objectos para roubar }\end{array}$ & Turistas, lojas \\
\hline $\begin{array}{l}\text { Aqui existem poucos turistas e lojas. Isso significa que } \\
\text { não existe dinheiro ou objectos para roubar }\end{array}$ & Ausência de turistas e lojas \\
\hline Este local tem má reputação & Má reputação do local \\
\hline Este local tem boa reputação & Boa reputação do local \\
\hline Este local apresenta boa visibilidade & Boa visibilidade \\
\hline Este local apresenta má visibilidade & Má visibilidade \\
\hline $\begin{array}{l}\text { Aqui existem muitos transportes. Isso "ajuda" os } \\
\text { carteiristas }\end{array}$ & Affordances dos transportes ${ }^{10}$ \\
\hline Outra (resposta livre) & Outra \\
\hline
\end{tabular}

$10 \mathrm{O}$ ambiente fornece-nos as oportunidades e os constrangimentos (um conjunto de affordances), que nós podemos usar como fonte de satisfação das nossas necessidades (Gibson, 1979). Affordances são os recursos ecológicos de um ponto de vista funcional. A forma como usamos o ambiente dependerá das nossas necessidades e interesses, valores e aspirações (Moser \& Uzzell, 2003). Desta forma, o ambiente pode proporcionar-nos tantas affordances quantas as intenções, incluindo as intenções criminais. 
A análise inferencial Bayesiana (Bernard, 2000; Bernard, 2003) permite, para as taxas de associação, determinar, a posteriori, (com uma garantia de 95\%) se cada uma delas está sobre- ou sub-representada relativamente à independência de duas modalidades, ou seja, se esta taxa de associação pode ser generalizada para a população global. Para uma associação positiva, a análise permite demonstrar se essa associação está sobre-representada ou se é impossível tirar alguma conclusão em relação à população global (nos casos em que não temos dados suficientes). Para uma associação negativa, demonstra se esta está sub-representada, ou se não é possível tirar alguma conclusão. De forma a simplificar a leitura dos resultados, será utilizado o termo "significativa" para designar sobre- ou sub-representações.

\section{Resultados}

Avaliação do risco de furto por carteirista em Alfama e na Praça do Comércio

Os resultados demonstram que, ao nível da nossa amostra, a relação entre as variáveis "Local" e "Avaliação do risco de furto por carteirista" é fraca $\left(\varphi^{2}=0,02, V^{2}\right.$ Cramer $\left.=2 \%\right)$. Neste sentido, em ambos os locais, mais de $60 \%$ dos inquiridos consideraram elevada a probabilidade de serem vítimas de furto por carteirista (Tabela 3). Através da análise inferencial Bayesiana, podemos ainda afirmar que não se verificou sub- ou sobre-representações de associações entre as modalidades das variáveis "Local" e "Avaliação do risco de furto por carteirista". Contudo, a análise das taxas de associação indica uma ligeira tendência para os respondentes, em Alfama, avaliarem o risco como "Muito baixo" e "Muito elevado", enquanto que os indivíduos entrevistados na Praça do Comércio avaliaram o risco como sendo "Provavelmente baixo", "Provavelmente elevado" e "Não sei". Isto é, os respondentes, em Alfama, avaliaram o risco de furto por carteirista com mais segurança, em comparação com os indivíduos na Praça do Comércio.

Tabela 3 - Taxas de associação entre o local e a avaliação do risco de furto por carteirista

\begin{tabular}{|c|c|c|c|c|c|c|}
\hline \multirow[b]{2}{*}{ Local } & \multicolumn{6}{|c|}{ Avaliação do risco de furto por carteirista } \\
\hline & Muito baixo & $\begin{array}{c}\text { Provavel- } \\
\text { mente baixo }\end{array}$ & Não sei & $\begin{array}{c}\text { Provavel- } \\
\text { mente elevado }\end{array}$ & $\begin{array}{cc}\text { Muito } & P c \\
\text { elevado } & \mathrm{gc}\end{array}$ & $\begin{array}{l}\text { Percenta- } \\
\text { gem total }\end{array}$ \\
\hline Alfama & $0,227(20,0 \%)$ & $-0,214(11,1 \%)$ & $-0,319(4,4 \%)$ & $-0,063(24,5 \%)$ & $0,082(40,0 \%)$ & $100 \%$ \\
\hline $\begin{array}{l}\text { Praça do } \\
\text { Comércio }\end{array}$ & $-0,217(12,8 \%)$ & $0,205(17,0 \%)$ & $0,305(8,6 \%)$ & $0,060(27,6 \%)$ & $-0,079(34,0 \%)$ & o) $100 \%$ \\
\hline
\end{tabular}


Uma explicação para este resultado pode ser o facto de a amostra de Alfama ser, maioritariamente, constituída por indivíduos que residiam nesse bairro (64,5\%), contrariamente ao que se verificou na amostra da Praça do Comércio $(2,1 \%)$. Paralelamente, como é visível através da Tabela 4, os respondentes que habitam fora de Lisboa estão sobre-representados na Praça do Comércio e sub-representados em Alfama. Por outro lado, os residentes de Lisboa estão sobre-representados em Alfama e sub-representados na Praça do Comércio.

Tabela 4 - Taxas de associação entre o local e a zona de residência (Lisboa, subúrbios de Lisboa ou outros locais)

\begin{tabular}{|c|c|c|c|c|}
\hline \multirow[b]{2}{*}{ Local } & \multicolumn{4}{|c|}{ Zona de residência } \\
\hline & Lisboa & $\begin{array}{c}\text { Subúrbios de } \\
\text { Lisboa }\end{array}$ & Outros locais & $\begin{array}{c}\text { Percentagem } \\
\text { total }\end{array}$ \\
\hline Alfama & $0,112 *(82,2 \%)$ & $-0,233(13,3 \%)$ & $-0,489 *(4,5 \%)$ & $100 \%$ \\
\hline Praça do Comércio & $-0,108 *(65,9 \%)$ & $0,223(21,3 \%)$ & $0,468 *(12,8 \%)$ & $100 \%$ \\
\hline
\end{tabular}

Este resultado permite-nos considerar a hipótese de que o grau de segurança na avaliação do risco de furto por carteirista pode estar relacionado com o local de residência dos inquiridos. De facto, os resultados encontrados suportam, parcialmente, esta suposição. Como é visível através da Tabela 5, a avaliação do risco como "Não sei" está sobre-representada entre os respondentes que vivem fora de Lisboa e sub-representada entre os residentes da capital. Paralelamente, a avaliação do risco como "Muito elevado" está sobre-representada entre os habitantes de Lisboa, enquanto que sub-representada entre os inquiridos que vivem nos subúrbios da cidade.

\section{Explicações verbais face à avaliação do risco de furto por carteirista}

Quando comparamos as explicações verbais atribuídas pelos inquiridos sobre a avaliação do risco de furto por carteirista, em Alfama e na Praça do Comércio, verificamos a existência de diferenças importantes.

Através das explicações atribuídas à avaliação do risco como sendo "Muito baixo", observamos que, em Alfama (Tabela 6), a categoria explicativa "Reactividade das outras pessoas aqui" está sobre-representada, enquanto que, na Praça do Comércio (Tabela 7), estão sobre-representadas as categorias explicativas "Não existem muitas pessoas aqui", "Este local é 
bastante vigiado (elevada presença da polícia/segurança)" e "Este local apresenta boa visibilidade".

Tabela 5 - Taxas de associação entre a avaliação do risco e a zona de residência (Lisboa, subúrbios de Lisboa ou outros locais)

\begin{tabular}{lcccc}
\hline \multirow{2}{*}{$\begin{array}{l}\text { Avaliação do risco de } \\
\text { furto por carteirista }\end{array}$} & \multicolumn{4}{c}{ Zona de residência } \\
\cline { 2 - 5 } & Lisboa & $\begin{array}{c}\text { Subúrbios } \\
\text { de Lisboa }\end{array}$ & Outros locais & $\begin{array}{r}\text { Percenta- } \\
\text { gem total }\end{array}$ \\
\hline Muito baixo & $-0,008(73,4 \%)$ & $-0,233(13,3 \%)$ & $\mathbf{0 , 5 3 3}(\mathbf{1 3 , 3 \% )}$ & $100 \%$ \\
Provavelmente baixo & $-0,167(61,5 \%)$ & $\mathbf{0 , 7 6 9 ( 3 0 , 8 \% )}$ & $-0,115(7,7 \%)$ & $100 \%$ \\
Não sei & $-\mathbf{0 , 5 4 9} *(\mathbf{3 3 , 3} \%)$ & $0,917(33,3 \%)$ & $\mathbf{2 , 8 3 3} * \mathbf{( 3 3 , 3 \% )}$ & $100 \%$ \\
Provavelmente & $-0,042(70,8 \%)$ & $\mathbf{0 , 1 9 8}(\mathbf{2 0 , 8 \% )}$ & $-0,042(8,4 \%)$ & $100 \%$ \\
elevado & $\mathbf{0 , 1 9 4} *(\mathbf{8 8 , 2} \%)$ & $\mathbf{- 0 , 4 9 3 * ( 8 , 8 \% )}$ & $-0,662(3,0 \%)$ & $100 \%$ \\
Muito elevado & & & & \\
\hline
\end{tabular}

Nota: * A associação é significativa (Análise inferencial Bayesiana)

Tendo em conta as explicações atribuídas à avaliação do risco como sendo "Provavelmente baixo", não se encontra uma sobre-representação de nenhuma categoria de explicação verbal em Alfama (Tabela 6). Contudo, ao nível desta amostra, os respondentes tendem a justificar as suas avaliações através das explicações "Reactividade das outras pessoas aqui", "As pessoas aqui são semelhantes a mim" e "Este local é bastante vigiado (elevada presença da polícia/segurança)". Relativamente à Praça do Comércio, verificou-se uma sobre-representação das categorias explicativas "Este local é bastante vigiado" e "Aqui existem muitos turistas e lojas. Isso significa dinheiro e objectos para roubar" (Tabela 7).

Quando o risco foi avaliado como "Não sei", a categoria "Outra" (respostas "Isto pode acontecer em qualquer lado", "Ninguém pode fazer nada") está sobre-representada em Alfama. Na Praça do Comércio não se verificou qualquer sobre-representação, porém, ao nível da nossa amostra, a maioria dos indivíduos tendem a explicar o risco de avaliação através das categorias "Passividade das outras pessoas aqui" e "Este local tem má reputação".

Tendo em conta as explicações atribuídas à avaliação do risco como "Provavelmente elevado", é de salientar a existência, em Alfama, de uma sobre-representação da categoria "Este local tem má reputação". No que respeita à Praça do Comércio, os indivíduos sobre-representaram a categoria "Aqui existem muitos turistas e lojas". 
Tabela 6 - Taxas de associação entre a avaliação do risco de furto por carteirista e as respectivas explicações verbais (Alfama)

\begin{tabular}{|c|c|c|c|c|c|}
\hline \multirow{2}{*}{$\begin{array}{l}\text { Explicações verbais atribuídas } \\
\text { à avaliação do risco }\end{array}$} & \multicolumn{5}{|c|}{ Avaliação do risco de furto por carteirista } \\
\hline & Muito baixo & $\begin{array}{l}\text { Provavel- } \\
\text { mente baixo }\end{array}$ & Não sei & $\begin{array}{l}\text { Provavelmen- } \\
\text { te elevado }\end{array}$ & $\begin{array}{c}\text { Muito } \\
\text { elevado }\end{array}$ \\
\hline Muitas pessoas & 0,025 & 1,179 & $-1,000$ & $-0,303$ & 0,010 \\
\hline Poucas pessoas & 1,871 & $-1,000$ & $-1,000$ & $-0,024$ & $-0,293$ \\
\hline Pessoas semelhantes a mim & 0,435 & 2,050 & $-1,000$ & $-0,024$ & $-0,293$ \\
\hline Pessoas diferentes de mim & 1,392 & $-1,000$ & $-1,000$ & $-1,000$ & 0,179 \\
\hline Ausência de deterioração & 0,435 & $-1,000$ & $-1,000$ & $-0,024$ & 0,061 \\
\hline Deterioração & $-1,000$ & $-1,000$ & $-1,000$ & 0,952 & 0,061 \\
\hline Passividade dos outros & 0,435 & $-1,000$ & 7,133 & $-1,000 *$ & 0,061 \\
\hline Reactividade dos outros & $2,588 *$ & 2,813 & $-1,000$ & $-1,000 *$ & $-0,558 *$ \\
\hline O local é vigiado & $-1,000$ & 2,813 & $-1,000$ & 1,440 & $-0,558 *$ \\
\hline O local não é vigiado & $-0,551$ & $-0,047$ & $-1,000$ & $-0,695 *$ & $0,437 *$ \\
\hline Turistas, lojas & $-0,448 *$ & $-1,000 *$ & 0,564 & 0,314 & $0,088 *$ \\
\hline Ausência de turistas e lojas & $-1,000$ & $-1,000 *$ & $-1,000$ & 3,880 & $-1,000 *$ \\
\hline Má reputação do local & $-0,448$ & $-1,000$ & $-1,000$ & $\mathbf{0 , 8 7 7 *}$ & $-0,048$ \\
\hline Boa reputação do local & 0,025 & 1,179 & $-1,000$ & $-0,651 *$ & 0,137 \\
\hline Boa visibilidade & 0,000 & 0,000 & 0,000 & 0,000 & 0,000 \\
\hline Má visibilidade & $-1,000$ & $-1,000$ & $-1,000$ & 2,253 & $-0,411$ \\
\hline Affordances dos transportes ${ }^{11}$ & 0,000 & 0,000 & 0,000 & 0,000 & 0,000 \\
\hline Outra & $-1,000$ & $-1,000$ & $19,333 *$ & $-1,000$ & $-0,116$ \\
\hline
\end{tabular}

Nota: * A relação é significativa (Análise inferencial Bayesiana)

Por fim, no que respeita à avaliação do risco como "Muito elevado", categorias como "Este lugar não é vigiado (reduzida presença da polícia/ segurança)" e "Aqui existem muitos turistas e lojas" estão sobre-représemtadas em Alfama, enquanto que as categorias "Existem muitas pessoas aqui: isso "ajuda" os carteiristas" e "Este lugar não é vigiado" estão sobre-representadas na Praça do Comércio.

$11 \mathrm{O}$ ambiente fornece-nos as oportunidades e os constrangimentos (um conjunto de affordances), que nós podemos usar como fonte de satisfação das nossas necessidades (Gibson, 1979). Affordances são os recursos ecológicos de um ponto de vista funcional. A forma como usamos o ambiente dependerá das nossas necessidades e interesses, valores e aspirações (Moser \& Uzzell, 2003). Desta forma, o ambiente pode proporcionar-nos tantas affordances quantas as intenções, incluindo as intenções criminais. 
Tabela 7 - Taxas de associação entre a avaliação do risco de furto por carteirista e as respectivas explicações verbais (Praça do Comércio)

\begin{tabular}{lccccc}
\hline $\begin{array}{l}\text { Explicações verbais } \\
\text { atribuídas à avaliação } \\
\text { do risco }\end{array}$ & \multicolumn{5}{c}{ Avaliação do risco de furto por carteirista } \\
\cline { 2 - 6 } & Muito baixo & $\begin{array}{c}\text { Provavel- } \\
\text { mente baixo }\end{array}$ & Não sei & $\begin{array}{c}\text { Provavelmen- } \\
\text { te elevado }\end{array}$ & $\begin{array}{c}\text { Muito } \\
\text { elevado }\end{array}$ \\
\hline Muitas pessoas & $-0,669 *$ & 0,055 & $-1,000 *$ & 0,120 & $\mathbf{0 , 2 1 0} *$ \\
Poucas pessoas & $\mathbf{7 , 2 8 6} *$ & $-1,000$ & $-1,000$ & $-1,000$ & $-1,000 *$ \\
Pessoas semelhantes a mim & $-1,000$ & $-1,000$ & $-1,000$ & $-1,000$ & 1,522 \\
Pessoas diferentes de mim & 0,000 & 0,000 & 0,000 & 0,000 & 0,000 \\
Ausência de deterioração & 1,762 & 0,758 & $-1,000$ & $-1,000$ & $-0,159$ \\
Deterioração & 0,000 & 0,000 & 0,000 & 0,000 & 0,000 \\
Passividade dos outros & $-1,000$ & $-1,000$ & $\mathbf{6 , 7 3 3}$ & $-1,000$ & 0,681 \\
Reactividade dos outros & 0,000 & 0,000 & 0,000 & 0,000 & 0,000 \\
O local é vigiado & $\mathbf{2 , 1 0 7 *}$ & $\mathbf{1 , 6 3 6} *$ & $-1,000$ & $-0,500$ & $-1,000 *$ \\
O local não é vigiado & $-1,000 *$ & $-0,623 *$ & $-1,000 *$ & $-0,143$ & $\mathbf{0 , 8 0 1} *$ \\
Turistas, lojas & $-0,693 *$ & $-0,805 *$ & $-0,141$ & $\mathbf{0 , 7 7 8} *$ & 0,121 \\
Ausência de turistas e lojas & $-1,000$ & $\mathbf{4 , 2 7 3} *$ & $-1,000$ & $-1,000$ & $-1,000 *$ \\
Má reputação do local & $-1,000$ & $-1,000 *$ & $\mathbf{3 , 6 4 0}$ & $-0,200$ & 0,513 \\
Boa reputação do local & $-0,247$ & 0,438 & 1,109 & $-0,273$ & $-0,083$ \\
Boa visibilidade & $\mathbf{3 , 9 7 1 *}$ & 1,109 & $-1,000$ & $-1,000 *$ & $-1,000 *$ \\
Má visibilidade & 0,000 & 0,000 & 0,000 & 0,000 & 0,000 \\
Affordances dos transportes ${ }^{12}$ & $-1,000$ & $-1,000$ & $-1,000$ & 1,000 & 0,261 \\
Outra & 0,184 & 1,260 & 2,314 & 0,143 & $-1,000 *$ \\
\hline
\end{tabular}

Nota: * A associação é significativa (Análise inferencial Bayesiana)

\section{Discussão}

\section{Avaliação do risco de furto por carteirista}

Neste estudo, não pudemos confirmar a nossa principal hipótese, visto que a população não avalia Alfama como um local de maior risco de furto por carteirista, relativamente à Praça do Comércio. No que diz

$12 \mathrm{O}$ ambiente fornece-nos as oportunidades e os constrangimentos (um conjunto de affordances), que nós podemos usar como fonte de satisfação das nossas necessidades (Gibson, 1979). Affordances são os recursos ecológicos de um ponto de vista funcional. A forma como usamos o ambiente dependerá das nossas necessidades e interesses, valores e aspirações (Moser \& Uzzell, 2003). Desta forma, o ambiente pode proporcionar-nos tantas affordances quantas as intenções, incluindo as intenções criminais. 
respeito à amostra da Praça do Comércio, podemos explicar a avaliação do risco de furto por carteirista como elevado, devido à falta de familiaridade das pessoas com o local (é importante recordarmos que a maior parte da nossa amostra não reside nesta área). E, como defendido por Moser \& Lidvan (1992), os sítios familiares são percepcionados pelos indivíduos como mais seguros do que sítios que não se conhecem.

A tendência de maior segurança na avaliação do risco de furto por carteirista pelos indivíduos entrevistados em Alfama, face àqueles que foram entrevistados na Praça do Comércio, pode ser explicada pela sua maior familiaridade com o local, uma vez que uma boa familiaridade espacial pode levar a níveis mais elevados de controlo pessoal e, consequentemente, ter um efeito de segurança nas avaliações do risco. Além de que uma maior familiaridade com os estímulos poderá fazê-los parecer relativamente mais previsíveis (Wickelgren, 1979) e uma boa familiaridade espacial está relacionada com uma maior confiança (Merry, 1981).

No entanto, em Alfama, os indivíduos familiarizados com o local tendem a avaliar como elevado o risco de furto por carteirista. Uma vez que Alfama é conhecido como um sítio onde vivem carteiristas, os nossos resultados podem ser explicados pelo facto de as pessoas estarem mais conscientes dos problemas sociais existentes no bairro (que na sua maioria são riscos para pessoas não residentes e estranhas ao bairro) e é provável que seja esse o motivo porque avaliam o risco de furto por carteirista como elevado.

Alfama é um bairro com uma identidade local e ligações sociais fortes, devido às suas particularidades espaciais e socioculturais, o que leva os seus habitantes a criarem um maior fortalecimento das ligações sociais (ver Robinson \& Wilkinson, 1995). Assim, as comunicações sociais intensas podem ser reforçadoras do medo do crime (ver Skogan \& Maxfield, 1981; Santinello \& Scacchi, 1998). Nas entrevistas realizadas verificámos que os casos de furto por carteirista cometidos dentro desta área são discutidos e comentados entre os residentes, servindo estas comunicações sociais para ampliar a preocupação social.

Também, de acordo com Albanesi (2003), o sentimento de comunidade tranquiliza os habitantes apenas de um modo emocional, mas não reduz o grau de preocupação pela seriedade dos problemas sociais. Isto significa que o julgamento da segurança de si próprio não depende somente dos sentimentos de pertença e integração numa comunidade, mas que está muito relacionado com as características e a representação do contexto de vida do indivíduo. 
Explicações verbais face à avaliação do risco de furto por carteirista em Alfama

Foi possível encontrar aspectos mais clarificadores nas explicações verbais dadas pelos sujeitos relativamente às avaliações do risco de furto por carteirista. Uma explicação adicional para a segurança na avaliação do risco nos respondentes de Alfama pode apoiar-se na confiança que estes sujeitos têm no seu ambiente social. De acordo com esta explicação, a nossa hipótese de o baixo controlo social informal ser devido à percepção de indícios de desorganização física e de deterioração em Alfama não pode ser corroborada. Com efeito, a relação entre os indícios de desorganização física objectivos e a percepção destes indícios não é linear (Taylor \& Hale, 1986), e é válida ao nível individual, mas não ao nível comunitário (Taylor, 2001). As respostas dadas em Alfama revelam uma percepção de controlo social informal elevado. Neste contexto, a explicação "Reactividade das outras pessoas aqui" (quando o risco é avaliado como "Provavelmente baixo" e "Muito baixo") pode ser interpretada como um apelo à eficácia social, cujas percepções elevadas têm um impacto na diminuição do medo do crime (Gibson et al., 2002).

Também, em Alfama, encontrámos uma categoria de explicação "As pessoas aqui são semelhantes a mim", o que nos leva a rejeitar a nossa hipótese sobre a percepção de a elevada heterogeneidade social estar relacionada com a avaliação do risco de furto por carteirista como elevado, neste bairro.

Face ao exposto, somos remetidos para a literatura existente sobre a relação entre ligações sociais e medo do crime. Tem sido demonstrado que os níveis baixos de medo do crime estão relacionados com factores como fortes ligações sociais (Hartnagel, 1979) e elevado sentimento de comunidade (Kim et al., 1997). Os resultados deste último estudo mostram que as características físicas do espaço não são preditores significativos da diferença entre o crime percebido e o crime objectivo. Este aspecto vai ao encontro dos resultados do nosso estudo, uma vez que os respondentes de Alfama não mencionam factores físicos dos locais para explicar as suas avaliações de reduzido risco de furto por carteirista.

Paralelamente, a categoria "Este local tem má reputação" está sobre-representada, quando o risco é avaliado como "Provavelmente elevado", o que pode explicar o facto de o risco de furto por carteirista ser considerado como elevado pelos sujeitos, principalmente pessoas familiarizadas com o lugar, que estão a par da reputação de Alfama, como argumentado previamente.

De referir igualmente que, sem qualquer surpresa, a incerteza máxima nas avaliações de risco neste local (resposta "Não sei") se ligam a interpretações fatalistas: "Ninguém pode fazer nada" e "Isto pode acontecer em 
qualquer lado", manifestando, assim, um grau mais baixo de controlo péssoal da possibilidade de ser alvo de furto por carteirista (como demonstrado anteriormente por Sautkina, 2004).

Finalmente, quando o risco de furto por carteirista é estimado como "Muito elevado", respondentes de Alfama referem-se a affordances positivos para os carteiristas (presença de pessoas com dinheiro) e um controlo social formal percebido baixo (falta de polícias e de vigilância).

\section{Explicações verbais face à avaliação do risco de furto por carteirista na Praça do Comércio}

De forma diferente, os entrevistados da Praça do Comércio justificaram as suas avaliações do risco, principalmente, segundo o ponto de vista da presença ou ausência de controlo social formal, affordances positivos ou negativos para os carteiristas (quer o risco de furto por carteirista seja avaliado como elevado, quer seja baixo). Especialmente, a percepção de affordances reflecte uma perspectiva mais individualista nas percepções pessoais dos sujeitos dos aspectos ambientais na Praça do Comércio: o grau de densidade social, a presença ou ausência de pessoas que transportem dinheiro ou objectos valiosos com eles.

A percepção do elevado grau de perspectiva desempenha um importante papel na avaliação de risco "Muito baixo" na Praça do Comércio, confirmando, assim, em parte, a nossa hipótese.

Além disso, uma percepção de elevada densidade social na Praça do Comércio (que, de facto, foi observada no nosso estudo) pode explicar as avaliações de risco como "Muito elevado" (ver Hunter \& Baumer, 1982; Sautkina, 2004).

Um resultado interessante é obtido quando os participantes demonstram um grau elevado de incerteza - avaliação do risco como "Não sei": esta é explicada quer pela falta de confiança no controlo social informal, quer pela má reputação do local. Insistimos que estas explicações não são dadas na avaliação do risco "Muito elevado" ou mesmo "Provavelmente elevado", mas antes no caso de incerteza de avaliação. Provavelmente, isto significa que alguns participantes podem ficar surpreendidos pelo facto de os entrevistadores lhes pedirem para estimar o risco de furto por carteirista num local como a Praça do Comércio, mas, no entanto, a questão fá-los pensar que, na verdade, o risco pode existir naquele local. De facto, e tal como propõem Ham-Rowbottom et al. (1999), os indivíduos não estão habituados a observar o seu ambiente numa perspectiva crítica e não gostam de pensar sobre os riscos de crimes. Paralelamente, a categoria "Passividade das outras pessoas aqui" pode ser explicada pela heterogeneidade social objectiva do local, o que leva um grande número de "estranhos" a passar 
por este local (devido à sua centralidade e proximidade de várias linhas de transporte), o que, por sua vez, pode originar sentimentos de incerteza e mesmo medo (ver Merry, 1981).

\section{Conclusões e Perspectivas}

Um dos resultados mais importantes deste estudo foi mostrar que as ligações sociais num local podem desempenhar um papel importante na avaliação do risco de furto por carteirista. Mais especificamente, em locais como Alfama, que podem ser percepcionados pelos seus habitantes como espaços semiprivados, a presença de fortes ligações sociais pode afastar e tornar menos importantes as características físicas para a avaliação do risco de furto por carteirista. Paralelamente, quando se trata de zonas que são frequentadas por muitos estranhos, em que, como qualquer outro lugar público, as ligações sociais e o controlo social informal são fracos (Taylor, 1987) e, logo, onde os indivíduos podem contar menos com a ajuda dos outros, as suas próprias percepções do meio físico e social podem tornar-se mais importantes para a avaliação do risco de furto por carteirista. Este princípio tem de ser comprovado em investigações relativas à avaliação do risco de outros crimes.

Alguns dos resultados obtidos neste estudo revelaram que a população pode ter algum tipo de representação ou estereótipo "ingénuo" acerca dos affordances para um carteirista. Neste sentido, e de acordo com Van der Wurf et al. (1989), os indivíduos têm ideias dos "espaços criminalizáveis", definidos como "a extensão em que uma situação conduz a actividades criminosas aos olhos de uma possível vítima, ou, de outra forma, quanto a situação facilita o criminoso" (p. 145). Este conhecimento geral dos "espaços criminalizáveis" deverá ser abordado em estudos futuros, dado que até hoje poucos se têm focado neste aspecto, embora tal análise pudesse contribuir para explicar melhor as avaliações do risco do crime e o fenómeno de vitimação.

Uma das limitações da nossa pesquisa é a tentativa (porém muito corrente) de estudar avaliações do risco do crime baseando as nossas interpretações na literatura dedicada principalmente ao medo do crime. Assim, uma distinção, ou uma aproximação entre estes dois fenómenos apresentam-se como necessárias, a ter em conta em futuros desenvolvimentos teóricos e da pesquisa.

Os estudos futuros devem também debruçar-se sobre as avaliações do risco de furto por carteirista, comparando a avaliação do risco das populações residentes, não residentes e dos turistas, porque os factores ambientais 
e psicossociais destas avaliações provavelmente difeririam, assim trazendo outras explicações às avaliações do risco deste tipo de crime.

\section{Referências}

Albanesi, C. (2003). Legami sociali e insicurezza in ambiente urbano: uno studio empirico. Bollettino di Psicologia Applicata, 240 (2), 15-25.

Appleton, J. (1975). The experience of landscape. London: Wiley.

Bernard, J.-M. (2000). BayACT, Bayesian analysis of contingency tables, local associations. Contingency table. Version 1.02 [Computer program]. Paris: Laboratoire de Psychologie Environnementale CNRS, Université Paris V.

Bernard, J.-M. (2003). Analysis of local asymmetric dependencies in contingency tables using the imprecise Dirichlet model. In J. M. Bernard, T. Seidenfeld, M. Zaffalon (Eds.), ISIPTA'03. Proceedings of the Third International Symposium on Imprecise Probabilities and Their Applications (pp. 46-61). Lugano.

Brantingham, P. J., \& Brantingham, P. L. (1991). Environmental criminology. Prospect Heights, IL: Waveland Press.

Brantingham, P. J., \& Brantingham, P. L. (1997). Understanding and controlling crime and fear of crime: Conflicts and trade-offs in crime prevention planning. In S. P. Lab (Ed.), Crime Prevention at a Crossroads. Cincinnati, OH: Anderson.

Brantingham, P. L., \& Brantingham, P. J. (1993). Nodes, paths and edges: considerations on environmental criminology. Journal of Environmental Psychology, $13,3-28$.

Chaguiboff, J. (1992). La représentation spatiale de l'insécurité. In Y. Bernard \& M. Segaud (Eds.), La ville inquiète (pp.67-73). Paris: Editions de l'Espace Européen.

Costa, A. F. (1999). Sociedade de bairro. Dinâmicas sociais da identidade cultural. Oeiras: Celta.

Donnelly, P. G. (1988). Individual and neighbourhood influences on fear of crime. Sociological Focus, 22 (1), 69-85.

Esteves, A. (1999). A criminalidade na cidade de Lisboa - uma geografia da insegurança. Lisboa: Colibri.

Félonneau, M.-L. \& Lannegrand-Willems, L. (2005). Normes adolescentes, normes adultes. Percevoir et juger les incivilités urbaines. Bulletin de psychologie, 58 (6), 480, 695-704.

Gibson, C., Zhao, J., Lovrich, N., \& Gaffney, M. (2002). Social integration, individual perceptions of collective efficacy, and fear of crime in three cities. Justice Quarterly, 19, 537-564.

Gibson, J. J. (1979). The ecological approach to visual perception. Boston: Houghton Mifflin.

Hall, E. T. (1966). The hidden dimension. New York: Doubleday. 
Ham-Rowbottom, K. A., Gifford, R., \& Shaw, K. T. (1999). Defensible space theory and the police: Assessing the vulnerability of residences to burglary. Journal of Environmental Psychology, 19, 117-129.

Hartnagel, T. F. (1979). The perception of fear of crime: Implication for neighbourhood cohesion, social activity and community affect. Social Forces, 58 (1), 176-193.

Hunter, A., \& Baumer, T. L. (1982). Street traffic, social integration, and fear of crime. Sociological Inquiry, 52 (2), 122-131.

Kelling, G. L., \& Moore, M. (1981). The Network Foot Patrol Experiment. Washington, D.C.: Police Foundation.

Kim, J. W., Mackin, J. R., \& Schweitzer, J. H. (1997). The relationship of community characteristics to actual and perceived crime in urban neighborhoods. Paper presented at the 27th Annual Meeting of Urban Affairs Association. Toronto, Canada.

Loewen, L. J., Steel, G. D., \& Suedfeld, P. (1993). Perceived safety from crime in the urban environment. Journal of environmental psychology, 13, 323-331.

Merry, S. (1981). Urban Danger: Life in a Neighborhood of Strangers. Philadelphia, PA: Temple University Press.

Moser, G., \& Lidvan, P. (1992). Environnement urbain et sentiment d'insécurité. In Y. Bernard \& M. Segaud (Eds.), La ville inquiète (pp. 53-66). Paris: Editions de l'Espace Européen.

Moser, G., \& Uzzell, D. (2003). Environmental Psychology. In T. Million \& M. J. Lerner (Eds.), Handbook of Psychology: Personality and Social Psychology. Vol. 5 (pp. 419-445). Hoboken, NJ, US: John Wiley \& Sons.

Nasar, J. L. (2000). The evaluative image of places. In W. B. Walsh, K. H. Craik \& R. H. Price (Eds.), Person-Environment Psychology: New directions and perspectives (2nd ed., pp. 117-168). Mahwah, NJ: Lawrence Erlbaum.

Nasar, J. L., \& Fisher, B. (1993). 'Hot spots' of fear and crime: A multi-method investigation. Journal of Environmental Psychology, 13, 187-206.

Nicolau, R. (1994). Análise multivariada da distribuição espacial de criminalidade em meio urbano. Dissertação de Mestrado não publicada. Instituto Superior Técnico, Lisboa.

Pain, R. (1993). Women's fear of sexual violence: explaining the spatial paradox. In H. Jones (Ed.), Crime and the Urban Environment. The Scottish experience (pp. 55-68). Avebury: Aldershot.

Perkins, D. D., Wandersman, A., Rich, R. C., \& Taylor R. B. (1993). The physical environment of street crime: Defensible space, territoriality and incivilities. Journal of Environmental Psychology, 13, 29-49.

Praça do Comércio. O seu a seu dono (1998). Boletim Lisboa Urbanismo. Retirado de http://ulisses.cm-lisboa.pt/data/002/003/001/artigo.php? $m l=3 \& x=b 2 a 4 p t$. $x m l$.

Relatório de Segurança Interna (2002). Lisboa: Ministério de Administração Interna. Retirado de www.mai.gov.pt/data/001/005/pdf/RelSegInt_2002.pdf. 
Robinson, D., \& Wilkinson, D. (1995). Sense of community in a remote mining town: Validating a Neighbourhood Cohesion Scale. American Journal of Community Psychology, 23, 137-148.

Rouanet, H., Le Roux, B., \& Bert, M.-C. (1987). Statistiques en sciences humaines: procédures naturelles. Paris: Dunod.

Santinello, M. \& Scacchi, L. (1998). Le dimensioni della paura del crimine. In M. Santinello, P. Gonzi \& L. Scacchi (Eds.), La paure della criminalità: Aspetti psicosociali di comunità. Milano: Giuffrè Editore.

Sautkina, E. (2004). Le vol à la tire: Facteurs environnementaux et psychosociaux de l'interaction voleur-victime. Thèse de Doctorat non-publiée. Université Paris 5 - René Descartes, Paris.

Silva, C. S. (2000). A complexidade familiar em Alfama. Retirado de www.aps.pt/ivcong-actas/acta164.pdf.

Skogan, W. G., \& Maxifield M. (1981). Coping with crime. Beverly Hills: Russel Sage Publishers.

Taylor, R. B. \& Hale, M. (1986). Testing alternative models of fear of crime. Journal of Law and Criminology, 77, 151-189.

Taylor, R. B. (1987). Toward an environmental psychology of disorder: Delinquency, crime, and fear of crime. In D. Stokols \& I. Altman (Eds.). Handbook of environmental psychology (vol. 1, pp. 7-40). New York: Wiley.

Taylor, R. B. (2001). Breaking away from broken windows. Baltimore neighborhoods and the nationwide fight against crime, grime, fear, and decline. Boulder, CO: Westview.

van der Wurf, A., van Staalduinen, L., \& Stringer, P. (1989). Fear of crime in residential environments: testing a social psychological model. Journal of Social Psychology, 129 (2), 141-160.

Wickelgren, W. A. (1979). Cognitive Psychology. New Jersey: Prentice-Hall.

Wilcox, P., Quisenberry, N., \& Jones, S. (2003). The built environment and community crime risk interpretation. Journal of Research in crime and delinquency, 40 (3), 322-345.

Wilson, J. Q., \& Kelling, G. (1982). Broken windows. Atlantic Monthly, March, 29-38 . 